\title{
Balanced Multiple Description Scalar Quantization
}

\author{
Ivana Radulovic and Pascal Frossard \\ Ecole Polytechnique Fédérale de Lausanne (EPFL) \\ Signal Processing Laboratories - LTS4 \\ CH-1015 Lausanne, Switzerland
}

\begin{abstract}
This paper tackles the problem of the generation of an arbitrary number of balanced descriptions with multiple description scalar quantization (MDSQ). We show how, with a very low complexity, we can vary the number of descriptions and the redundancy between them, in order to adapt to different channel characteristics. A comparison with state-of-the-art MDSQ schemes shows a better performance of our solution in terms of an average distortion at the receiver, which comes from the flexibility of our solution to better adapt to various lossy conditions.
\end{abstract}

\section{INTRODUCTION}

The goal of multiple description coding (MDC) is to build several representations (descriptions) of a source in such a way that a high quality reconstruction is achieved if all the descriptions are available, while this quality gracefully degrades in case some of them are missing. If, in addition, all descriptions have equal rates and if a combination of any $k$ out of $N$ descriptions induces equal distortions, we say that the descriptions are balanced.

Multiple description scalar quantization (MDSQ) is a popular technique for the generation of multiple descriptions [1]. The design of an MDSQ system generally follows two steps: a scalar quantization and an index assignment, which maps the quantized values to $\mathrm{N}$-tuples of quantization indices $\left(i_{1}, i_{2}, \ldots, i_{N}\right)$, corresponding to the different descriptions. Equivalently, we can think of the MDSQ as having $N$ scalar quantizers, where each of them is used to produce one of the descriptions, as depicted on Figure 1.

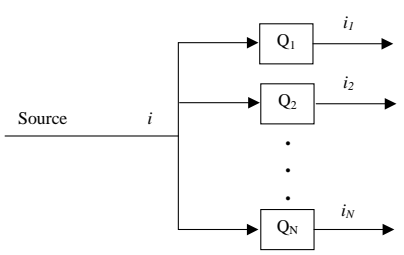

Fig. 1. Multiple Description Scalar Quantization

Most of the MDSQ schemes proposed in the literature work for $N=2$ descriptions only [2]-[6] and are not easily extendable to an arbitrary number of descriptions due to complexity reasons. At the same time, it has been suggested in the literature that, as the loss rate increases, it becomes more beneficial to have more than two descriptions, [7], [8]. Yet, much less work has been reported in the literature for an arbitray number of descriptions. An MDSQ design for three descriptions that combines staggered quantization with unequal error protection has been proposed in [9]. However, this solution only guarantees that the descriptions are asymptotically balanced, and is not easily extendable to more than three descriptions. The author in [10] proposes a multistage MDSQ design for a dyadic number of descriptions based on [1], where each stage doubles the number of descriptions. The problem of the design of an arbitrary number of descriptions has also been addressed from the combinatorial point of view in [11].

In this paper we tackle the problem of designing an arbitrary number of descriptions, while trying to keep the design complexity very low. The proposed scheme offers the possibility to adapt to loss probability, and rate constraints, by playing with both the number of descriptions, and the rate of each of them, in order to minimize the average distortion. The comparison with the existing MDSQ solutions for two and three descriptions shows that our scheme gives smaller average distortion for most of the considered scenarios.

\section{BALANCED MDSQ}

In this section, we consider the balanced MDSQ in more details. As our main objective is to build a low complexity encoding scheme, we consider only convex quantization cells, which might however not always be optimal in MDC [1]. We assume that our source is uniformly distributed on the interval $[0,1]$. Each of the $N$ descriptions has a rate of $R_{i}=R$ bits/symbol, $i=1, \ldots, N$, which further leads to a total rate of $R_{t o t}=N R$. We assume that the rate is measured as the average codeword length at the output of each quantizer. We further assume that the distortion is measured by the mean squared error (MSE).

In order to achieve balanced rates, the proposed solution has to rely on $N$ side quantizers with the same sets of bins $\left\{\Delta_{j}\right\}$. This is a sufficient condition since $R_{i}=-\sum_{j} \Delta_{j} \log _{2} \Delta_{j}$, where $\Delta_{j}$ is the size of bin $j$. Moreover, all the side distortions $D_{i}$ are also equal since they are given by $D_{i}=\int_{0}^{1} p(x)(x-\widehat{y})^{2} d x=\sum_{j} \frac{\Delta_{j}^{3}}{12}$, where $\widehat{y}$ are the corresponding centroids of quantization bins. In addition, the partial distortions $D_{K}$, induced by receiving any set of $k$ descriptions $(2 \leq k<N)$ should also be equal in a balanced MDSQ scheme. A sufficient condition for balanced partial distortions is that the combination of any $k$ descriptions in a partial decoder also provides the same sets of bins, for all the partial decoders. Finally, since the uniform quantization minimizes 
distortion for uniform sources, a proper MDSQ design should result in a uniform quantization of the source central quantizer when all $N$ descriptions are combined together.

\section{GENERIC QUANTIZER CONSTRUCTION}

We now propose a generic and intuitive solution for the design of side quantizers given the above constraints. Assume first that the side quantizers are built on $N$ blocks, $B_{1}, \ldots, B_{N}$, that represent combinations of quantization bins. We have a full freedom in their design, as long as two constraints are satisfied: 1) they are of equal length and 2) the combination of all of them (the union of the quantization bins from all the blocks) results in a uniform quantizer with step-size $\delta$. The former condition is introduced due to simplicity reasons, while the latter one ensures a uniform quantization in the central quantizer. We can build $N$ scalar quantizers with the blocks $B_{1}, \ldots, B_{N}$ or any multiple of them, but under the constraint that each quantizer contains the same sets of blocks, which ensures balance in rates and side distortions.

To illustrate this, we show two examples on Figure 2, where the number of blocks/descriptions is three. The left figure shows three blocks, where second and third blocks contain the same set of bins $\{\delta, 2 \delta, 3 \delta\}$, but arranged differently, while the first one contains the set $\{\delta, 5 \delta\}$. While the combination of these three blocks in different side quantizers results in a uniform quantizer with step size $\delta$ (where by combination we mean taking a union of quantization levels of these blocks), we can see that no pair of blocks shares the same quantization levels, except at the block boundaries. Therefore, we can think of this case as of a "low-redundancy" case, where each additional block strictly refines the quantization bins of the partial or central quantizers when these blocks belong to different descriptions. On the other hand, example (b) shows another extreme case, where all the blocks are uniform quantizers with step-sizes $\delta$. This is the case with highest redundancy.

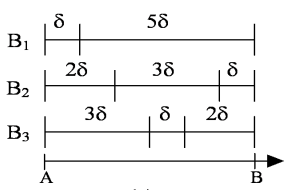

(a)

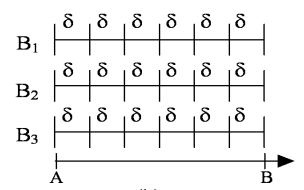

(b)

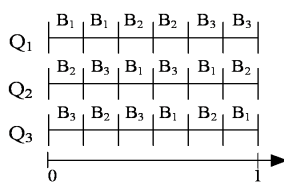

(c)
Fig. 2. Some designs of blocks and quantizers which guarantee $N=3$ balanced descriptions: (a) low redundancy case, (b) highest redundancy case, (c) structure of quantizers $Q_{1}-Q_{3}$ with $3 !=6$ blocks.

We analyze now in more details the conditions that permit to achieve balanced descriptions with a block construction. Denote the number of occurrences of each block per quantizer $\operatorname{occ}(N)$. Now, the side quantizer $Q_{i}$, containing some combination of the blocks, can be thought of an array which is filled in with the elements from $\left\{B_{1}, \ldots, B_{N}\right\}$. The size of such an array is clearly $N \cdot \operatorname{occ}(N)$. In addition, we are looking for a design that also ensures balanced partial distortions. A trivial solution can be obtained with all possible cyclic permutations of blocks $B_{1}, \ldots, B_{N}$, and by distributing the blocks of each permutation sequentially in each description. This ensures that all the blocks are present for any value of the source. There are $N$ ! permutations of $N$ blocks and therefore, in the worst case, we can achieve a balanced scheme when each side quantizer is a set of $N$ ! blocks. This also means that there will be $\operatorname{occ}(N)=\frac{N !}{N}=(N-1)$ ! occurrences of each of the blocks $B_{1}, \ldots, B_{N}$ in all the quantizers. Moreover, any $k$ quantizers taken together give $\frac{N !}{\left(\begin{array}{l}N \\ k\end{array}\right)}$ of each of the possible $k$-combination of $N$ blocks.

An example when $N=3$ is shown on Figure 2(a). There are $3 !=6$ permutations of blocks $B_{1}-B_{3}$. We can see that all the quantizers contain two occurrences of each of the blocks $B_{1}-B_{3}$. Next, we can notice that any two quantizers combined together give all the possible 2-combinations of three blocks: $\left\{B_{1} B_{2}, B_{1} B_{3}, B_{2} B_{3}\right\}$. Finally, we see that all quantizers combined together result in a uniform quantizer since each column in a matrix contains all the blocks $B_{1}-B_{3}$, which guarantees the all- $\delta$ property by construction. Therefore, all conditions for three balanced descriptions with these quantizers are met.

However, although simple and straightforward, the solution with all the $N$ ! permutations is rather naive and definitely not minimal. For example, it can be seen that when $N=3$, the minimal solution requires only three out of possible six permutations. Before deriving the minimal number of block permutations for balanced descriptions, we give another example when the number of description $N=4$ (see Figure 4). It represents the structure with the minimal necessary number of permutations for achieving four balanced descriptions. As we can see, only 12 (rather than $N !=4 !=24$ ) permutations are needed. On this example, we can also notice that any two quantizers combined together contain the same number of all the possible 2-combinations of four blocks. Namely, there are $\left(\begin{array}{l}4 \\ 2\end{array}\right)=6$ such 2-combinations: $\left\{B_{1} B_{2}, B_{1} B_{3}, B_{1} B_{4}, B_{2} B_{3}, B_{2} B_{4}, B_{3} B_{4}\right\}$ and we can see that each of them is repeated twice in the proposed design. Further on, any three quantizers contain all the 3-combinations of four blocks. There are $\left(\begin{array}{l}4 \\ 3\end{array}\right)=4$ such combinations: $\left\{B_{1} B_{2} B_{3}, B_{1} B_{2} B_{4}, B_{1} B_{3} B_{4}, B_{2} B_{3} B_{4}\right\}$ and, as can be seen from Figure 3 , all of them are repeated three times with this solution. Finally, all the quantizers taken together give a uniform quantizer, since each column contains all the blocks $B_{1}, \ldots, B_{4}$.

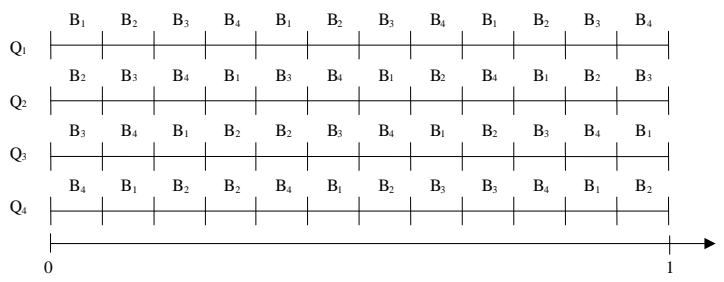

Fig. 3. Minimal solution for scalar quantizers when $N=4$.

Now we show how to determine the minimal number $\operatorname{occ}(N)$ of occurrences of blocks $B_{1}, \ldots, B_{N}$, for an arbitrary number of descriptions $N$. 
Theorem 1. Assume we build $N$ quantizers with blocks $B_{1}, \ldots, B_{N}$. The minimal number of occurrences of each block per quantizer that ensures balanced descriptions is:

$$
\operatorname{occ}(N)_{\min }=\frac{\operatorname{LCM}\left(\left(\begin{array}{c}
N \\
1
\end{array}\right),\left(\begin{array}{c}
N \\
2
\end{array}\right), \ldots\left(\begin{array}{c}
N \\
N
\end{array}\right)\right)}{N},
$$

where LCM denotes the least common multiple.

Proof: Our quantizers design should ensure that any combination of $k \leq N$ quantizers gives the same number of all the possible k-combinations of blocks $B_{1}, \ldots, B_{N}$. For each $k$, there are $\left(\begin{array}{l}N \\ k\end{array}\right)$ such $k$-combinations. The minimal number of occurrences of blocks $B_{1}, \ldots, B_{N}$ in each quantizer can therefore be taken as the minimal number that is a factor of all the numbers $\left\{\left(\begin{array}{c}N \\ k\end{array}\right)\right\}_{k=1}^{N}$, divided by $\mathrm{N}$ (the number of blocks). Clearly, the smallest such number is the least common multiple of all $\left\{\left(\begin{array}{c}N \\ k\end{array}\right)\right\}$, given by Eq. (1).

Under the above design constraints, the minimal achievable rate with block permutation is obtained if all the blocks are equal, all with one bin of size $\delta$. The minimal rate can be calculated as $\log _{2}\left(N \cdot \operatorname{occ}(N)_{\min }\right)$, since $\delta=\frac{1}{N \cdot o c(N)_{\min }}$. The minimal rate is therefore $1 \mathrm{bit} / \mathrm{symbol} / \mathrm{description}$ for $N=$ 2 . Further on, the minimal rate is $3.58 \mathrm{bits} / \mathrm{symbol} /$ description for $N=4$, and $5.91 \mathrm{bits} / \mathrm{symbol} / \mathrm{description}$ for $N=6$.

The flexibility we have in our quantizers design comes at a high cost since the minimal achievable rates per description increase greatly as we increase the number of descriptions. Therefore, we present in the next section one solution to reduce the minimal rate, and yet to keep the descriptions balanced. As we will see, we can simplify our design and reduce the number of permutations when we put additional constraints on the construction of the blocks $B_{1}, \ldots, B_{N}$.

\section{SINGLE BLOCK QUANTIZERS}

So far, we have allowed an arbitrary structure of the blocks, as long as their combination results in a uniform scalar quantization. This freedom imposes constraints on the minimal number of occurrences of these blocks in each quantizer, which further dictates the minimal achievable rates. In this section, we show how we can reduce the rates if we impose additional constraints on the block structures.

We propose to decrease the coding rate by relaxing the constraints on block permutations, and by imposing rules on the design of the blocks themselves. We consider a scheme where each side quantizer is built on one single block of quantization bins. In this case, having all the blocks of the different quantizers contain same sets of bins, and where any $k$ blocks combined together also provide equal sets of bins remains a sufficient condition for balanced descriptions. It is clear that imposing these constraints narrows the set of initial solutions, but it also reduces the rates that can be achieved by block permutations.

Now we show one possible solution, where the bin sizes obtained by combining any $k \geq 2$ side quantizers can only take two values: $a \delta$ or $\delta$, where $a$ is an integer, with $a \geq 2$. In addition, we assume that every additional side quantizer refines one of the remaining bins $a \delta$ in the joint quantizer, to $a$ bins of size $\delta$. This basically means that the combination of $k \geq 2$ side quantizers gives $N-k$ bins of size $a \delta$ and the bins of size $\delta$, which finally imposes $N-1$ coarse bins per quantizer. In our design, we permit $N-2$ bins of size $a \delta$ and one bin of size $b \delta$, per side quantizer, where $\mathrm{b}$ is an integer, $b \geq a$. Finally, we propose the quantizer $Q_{i}, i=2, \ldots, N$, to be a cyclic shift of $Q_{i-1}$ by a certain structure. Thus, we only need to define the structure of $Q_{1}$, while the remaining ones will be obtained straightforwardly.

Before getting in more details about the quantizer design, we give few examples that explain our intuition. Figure 4(a) shows the simplest way of obtaining $N=4$ balanced descriptions, which corresponds to the case when $a=b$. We start from a uniform block with bins $a \delta$ and we subsequently divide the last bin into $a$ bins of size $\delta$. The structure of the remaining $N-1$ side quantizers is obtained by cyclic shifting by the bin $a \delta$. It is not difficult to verify that the above-mentioned constraints for balanced descriptions are satisfied.

Figure 4(b) shows an example for $N=5$ descriptions, where the last two bins $a \delta(a=4)$ are merged to a bin $2 a \delta$ and subsequently split into a bin $b \delta(b=5)$ and three bins $\delta$. Cyclic permutation of such a structure, by a bin $a \delta$, provides the remaining side quantizers. Shifting the structure of $Q_{4}$, which starts with a bin $b \delta$, by a length $a \delta$, leaves a part of size $\delta$ at the beginning of the structure of $Q_{5}$, which therefore has to be added to all the other blocks to compensate for edge effects and still guarantee balanced descriptions. From this example it is also clear that $b$ cannot exceed $a+1$. Otherwise shifting a bin $b \delta$ by $a \delta$ would leave some of its parts that have a length larger than $\delta$. This would further violate the condition that a combination of any $k$ blocks gives either bins $a \delta$ or $\delta$. However, we can allow for an arbitrary $b$ if we place $b-a-1$ bins $\delta$ after each bin $a \delta$. We can still guarantee balanced descriptions, by shifting each side quantizer by a bin $a \delta$ and $b-a-1$ bins $\delta$, as can be seen on Figure 4(c) for $N=4, a=3$ and $b=7$.

Increasing the shifting structure by $b-a-1$ bins further implies having $2 \cdot(a+b-a-1)-b=b-2$ of bins $\delta$ after the bin $b \delta$. Finally, one more bin $\delta$ has to be added again to compensate for the edge effect, which in total makes $b-1$ bins $\delta$ after the bin $\delta$. To summarize, the generic structure of the side quantizer $Q_{1}$ is depicted on Figure 5. It is intuitively clear that the optimal MDSQ design, i.e., the choice of parameters $N, a$ and $b$ depends on the channel characteristics, as shown in the next section.

It can be seen that the above design guarantees balanced descriptions. Firstly, since the side quantizers rely on the same sets of bins, the rates and side distortions remain balanced. Secondly, any $k \geq 2$ blocks combined together give $a(k-1)+2 b-a-1$ bins $\delta$ and $(N-k)$ bins $a \delta$. This guarantees balanced partial distortions. Finally, all of them combined together result in the uniform quantizer with stepsize $\delta$. Thus, our descriptions remain balanced for any choice of parameters $N, a$ and $b$.

We can vary the rates of our descriptions by choosing dif- 


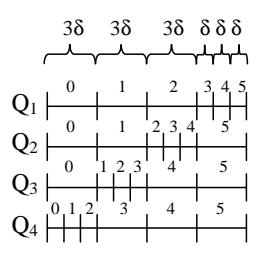

(a) $N=4, a=b=3$

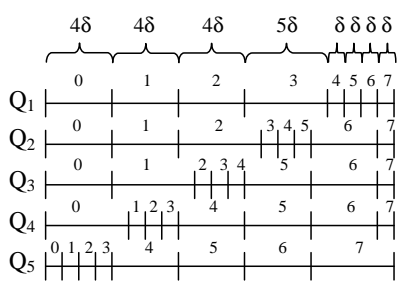

(b) $N=5, a=4, b=5$

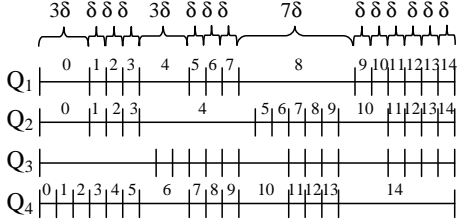

(c) $N=4, a=3, b=7$

Fig. 4. Possible designs of quantizers which guarantee balanced descriptions.

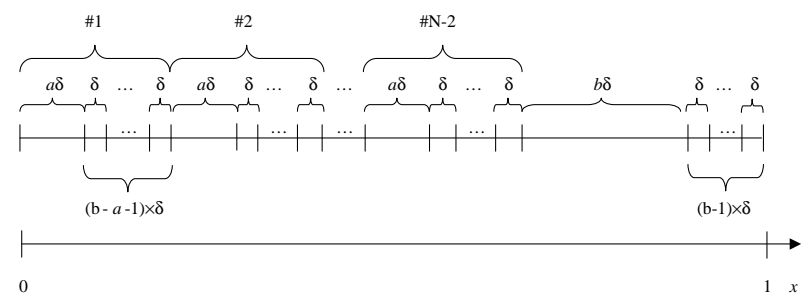

Fig. 5. The structure of the quantizer $Q_{1}$.

ferent combinations of $N, a$ and $b$ and/or by simple repeating of the quantizer structure $m$ times, where $m$ is an integer, $m \geq 1$. After we take into account these two ways of varying the rate, we can write: $\delta=\frac{1}{m(a(N-2)+2 b-1)}$ and the following expressions for rates, side distortions $D_{i}$ and partial distortions $D_{K}$ :

$$
\begin{gathered}
R_{i}=-\log _{2} \delta-\frac{b \log _{2}(b)+(N-2) a \log _{2}(a)}{a(N-2)+2 b-1} \\
D_{i}=\frac{(N-2) a^{3}+b^{3}+b-1}{12} m \delta^{3} \\
D_{K}=\frac{(N-k) a^{3}+(k-1) a+2 b-a-1}{12} m \delta^{3}, k \geq 2
\end{gathered}
$$

Finally, the minimal rate that can achieved by the block permutation scheme for a given $N$ can be obtained by putting $a=b=m=1: R_{\text {min }}(N)=\log _{2}(N)$. This is significantly lower than $\log _{2}\left(N \cdot \operatorname{occ}(N)_{\min }\right)$. For example, when $N=6$, the minimal rate drops from initial 5.91 to 2.59 bits/symbol/description. Therefore, by putting more stringent constraints on the blocks design, we can achieve smaller rates.

\section{Simulation Results}

We evaluate now the performance in terms of average distortion, and we compare it to state-of-the-art solutions. Suppose that our MDSQ scheme is applied to each source symbol, drawn from a uniform distribution, which gives us $N$ new encoded symbols, considered to be separate descriptions. Each description is then sent over a lossy channel and can be lost with probability $p$, independently of the other symbols.. Since the descriptions are balanced, the reconstruction distortion depends only on the number of received descriptions, but not on which particular set is received. If all of them are received, which happens with the probability $(1-p)^{N}$, the signal is reconstructed with the lowest, central distortion, $D_{N}$. If $k \leq N$ descriptions are received - this happens with the probability $\left(\begin{array}{l}N \\ k\end{array}\right) p^{N-k}(1-p)^{k}$ - the signal is reconstructed with the corresponding side or partial distortion $D_{K}$. The most severe case corresponds to losing all the descriptions, which happens with the probability $p^{N}$. In that case, the receiver can only guess what is sent by the sender and the corresponding distortion is equal to the variance of the source, $\sigma^{2}$. Therefore, we can write the average distortion in the presence of losses in the following way:

$$
D_{a v}=\sum_{k=0}^{N-1}\left(\begin{array}{l}
N \\
k
\end{array}\right) p^{k}(1-p)^{N-k} D_{N-k}+p^{N} \sigma^{2}
$$

We can see that $D_{a v}$ depends on the number of descriptions, rates of descriptions and the probability of failure. We consider the average distortion as a good metrics, which combines the performance of the proposed scheme on one side, and the channel characteristics on the other side.

To find the optimal parameters for our solution, we formulate the following problem:

Problem formulation: Given the probability $p$ of losing a description, and the total rate $R_{\text {tot }}$, find the parameters $N$, $m$, $a$ and $b$ that minimize the average distortion, $D_{a v}$, given in Eq.(5).

The solution to this problem is given in Table 1, which shows the optimal parameters for the total bitrates of 5 and 25 bits/symbol. We can see that, for a given rate $R_{t o t}$, with the increase of packet loss ratio, the optimal number of descriptions increases and the rate of each description decreases. In addition, the redundancy $\rho=R_{\text {tot }}-R^{*}$ also increases, where $R^{*}$ represents the rate required by a single description coder to achieve the same central distortion as in the multiple description case. This is not a surprising result, since as the losses tend to increase, it makes sense to send more descriptions and to make them more redundant, with the hope that at least one of the descriptions is received and the most important information is recovered. We can also see that the parameters $a$ and $b$ decrease as $p$ increases. This can be explained by the fact that the decrease of these parameters directly influences the increase in redundancy. Figure 6 shows the minimal achievable average distortions as functions of $p$, for different total bitrates. We can see that the 
case when the rate budget is small is much more sensitive to losses of descriptions. This is due to the fact that we can produce fewer descriptions at lower rates, but also because these descriptions are necessarily less redundant. Losing one of them therefore causes higher increase in distortion than in the case of more redundant descriptions.

\begin{tabular}{|c|c|c|c|c|c|}
\hline & $R_{t o t}[$ bits/sym.] & $p=10^{-3}$ & $p=10^{-2}$ & $p=0.1$ & $p=0.3$ \\
\hline $\bar{N}$ & \multirow{5}{*}{5} & 2 & 2 & 2 & 3 \\
\hline $\mathrm{m}$ & & 1 & 1 & 1 & 1 \\
\hline $\mathrm{a}$ & & 1 & 1 & 1 & 1 \\
\hline \multirow{2}{*}{$\begin{array}{l}\mathrm{b} \\
\rho\end{array}$} & & 10 & 10 & 3 & 2 \\
\hline & & 0.751 & 0.751 & 1.81 & 2.5 \\
\hline $\bar{N}$ & \multirow{5}{*}{25} & 3 & 3 & 4 & 6 \\
\hline \multirow{4}{*}{$\begin{array}{c}\mathrm{m} \\
\mathrm{a} \\
\mathrm{b} \\
\rho\end{array}$} & & 32 & 48 & 13 & 1 \\
\hline & & 39 & 12 & 5 & 3 \\
\hline & & 40 & 13 & 6 & 7 \\
\hline & & 13.11 & 14.2 & 16.9 & 19.78 \\
\hline
\end{tabular}

TABLE I

OPTIMAL PARAMETERS $N, m, a$ AND $b$ THAT MINIMIZE THE AVERAGE DISTORTION IN THE PRESENCE OF LOSSES.

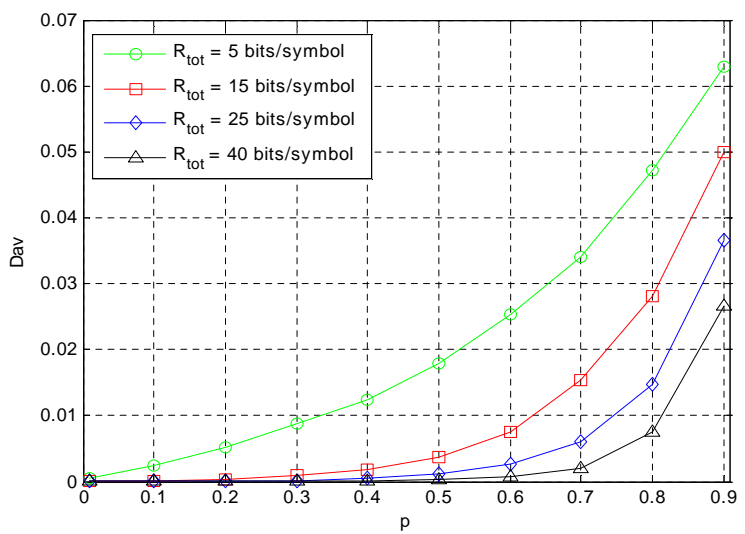

Fig. 6. Minimal achievable average distortion, as a function of different total bitrates and probability of loss.

We finally consider how our MDSQ scheme compares to the staggered schemes proposed in the literature, for two and three descriptions, in terms of minimal achievable average distortions as functions of a loss probability. In the staggered MDSQ for two and three descriptions, the side quantizers are offset one half and one third of the quantization step size respectively to one another. We consider the bitrate of $R_{t o t}=25$ bits/symbol. Figure 7 illustrates this comparison. We first see that the single description coding scheme performs worst in the whole range of considered probabilities of losses. Clearly, it does not make sense to use all the available bits for pure source coding without any added channel redundancy. The staggered scheme for two descriptions performs best in a very narrow range of losses, thanks to a smaller redundancy than in three-description coding schemes. Finally, we see that our solution performs best when $p$ exceeds $3 \cdot 10^{-4}$, and that it always outperforms the staggered MDSQ for three descriptions thanks to its better adaptivity to different lossy constraints.

\section{CONCLUSIONS}

In this paper, we have shown a simple and effective MDSQ scheme that can construct an arbitrary number of balanced

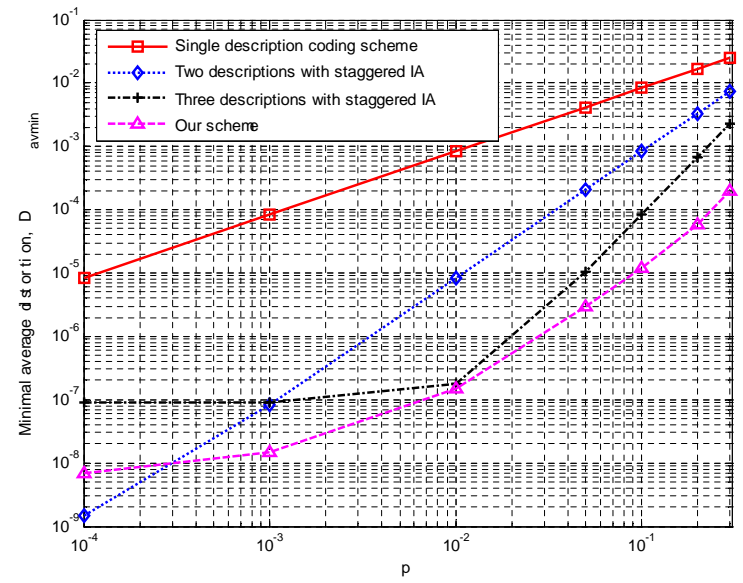

Fig. 7. Minimal achievable average distortions for our, single and twoand three-description coding schemes with staggered index assignment for $R_{\text {tot }}=25$ bits/symbol.

descriptions with low rate compared to generic block permutation schemes. We have shown that we can adapt to different channel characteristics by varying the number of descriptions and redundancy in our design. Finally, a comparison with state-of-the-art MDSQ schemes has demonstrated an improved performance of our solution in terms of the average distortion, thanks to the flexibility to optimally tune the parameters of our scheme to different lossy environments.

\section{REFERENCES}

[1] V.A. Vaishampayan, "Design of multiple description scalar quantizers," IEEE Trans. on Information Theory, vol. 39, no. 3, pp. 821-834, May 1993.

[2] C. Tian; and S.S. Hemami, "Universal multiple description scalar quantization: analysis and design ," IEEE Trans. on Information Theory, vol. 50, no. 9, pp. 2089-2102, Sept. 2004.

[3] C. Tian and S.S. Hemami, "A special class of multiple description scalar quantizers," in Proceedings of IEEE Information Theory Workshop, Oct. 2004, pp. 135-140.

[4] J. Cardinal, "Entropy-constrained index assignments for multiple description quantizers," IEEE Trans. on Signal Processing, vol. 52, no. 1, pp. 265-270, Jan. 2004.

[5] D. Muresan and M. Effros,, "Quantization as histogram segmentation: globally optimal scalar quantizer design in network systems," in Proceedings of the IEEE Data Compression Conference, Mar. 2002, pp. 302-311.

[6] S. Dumitrescu, X. Wu and G. Bahl, "Fast algorithms for optimal twodescription scalar quantizer design," in Proceedings of the IEEE Data Compression Conference, Mar. 2004, pp. 42-51.

[7] I. Radulovic and P. Frossard," "Fast index assignment for balanced n-description scalar quantization," in Proceedings of the IEEE Data Compression Conference, Mar. 2005, p. 474.

[8] J. Ostergaard, J. Jensen and R. Heusdens, "n-Channel entropyconstrained multiple description lattice vector quantization," IEEE Trans. on Information Theory, vol. 52, no. 5, pp. 1956- 1973, May 2006.

[9] C. Tian and S.S. Hemami,, "Sequential design of multiple description scalar quantizers," in Proceedings of the Data Compression Conference, Mar. 2004, pp. 32-41.

[10] J. Cardinal, "Multistage index assignments for M-description coding," in Proceedings of IEEE International Conference on Image Processing, vol. 3, Sept. 2003, pp. 249-252.

[11] T.Y. Berger-Wolf and E.M. Reingold, "Index assignment for multichannel communication under failure," IEEE Trans. on Information Theory, vol. 48, no. 10, pp. 2656 - 2668, Oct. 2002. 